\title{
Evaluation of The Virtual Cells Software: a Teaching Tool
}

\author{
C.C.P. da Silva; N.F. dos Santos; L.M. Beltramini \\ Centro de Biotecnologia Molecular Estrutural (CBME/CEPID/FAPESP) - Instituto de Física de São Carlos - \\ USP, São Carlos, SP. e-mail: neusafs@if.sc.usp.br
}

Studies show that the use of games and interactive materials at schools is a good educational strategy, motivating students to create mental outlines and developing the reasoning and facilitating the learning. In this context, the Scientific Dissemination Coordination of the Center for Structural Molecular Biotechnology (CBME), developed a series of educational materials destined to the elementary and high schools, universities and general public. Among these, we highlighted the Virtual Cells software that was developed with the aim of helping in the understanding of the basic concepts of cell types, their structures, organelles and specific functions. Characterized by its interactive interface, this software shows eukaryotes and prokaryotes cells images, where organelles are shown as dynamic structures. In addition, it presents exercises in another step that reinforce the comprehension of $\mathrm{Cy}$ tology. A speaker narrates the resources offered by the program and the necessary steps for its use. During the stage of development of the software, students and teachers of public and private high schools from São Carlos city, São Paulo State, were invited to register their opinions regarding the language and content of the software in order to help us in the improvement of it. After this stage, the Scientific Dissemination Coordination of CBME organized a series of workshops, where 120 individuals evaluated the software (students and teachers of high school and others undergraduate students). For this evaluation, a questionnaire was elaborated based on the international current literature in the area of sciences teaching and it was applied after the interactive section with the software. The analysis of the results demonstrated that most of the individuals considered the software of easy handling, having an accessible language, supporting the software as an education tool that is capable to facilitate the learning of the fundamental concepts about the theme. Other workshops are programmed to happen with participants from different educational institutions of São Carlos city, with the goal to broaden our sample. Supported by CNPq and FAPESP (CBME/CEPIDS, Center of Research, Innovation and Dissemination) 